\title{
Incidence and Predictors of Pregnancy among Women on ART in Debre Markos Referral Hospital, Northwest Ethiopia: A Five-Year Retrospective Cohort Study
}

\author{
Maru Meseret, ${ }^{1}$ Alemayehu Shimeka, ${ }^{2}$ and Alemayehu Bekele ${ }^{3}$ \\ ${ }^{1}$ Amhara National Regional State Health Bureau, East Gojjam Zonal Health Department, Bahir-Dar, Ethiopia \\ ${ }^{2}$ Department of Epidemiology and Biostatistics, College of Medicine and Health Sciences, University of Gondar, Gondar, Ethiopia \\ ${ }^{3}$ Ethiopian Public Health Association, Addis Ababa, Ethiopia \\ Correspondence should be addressed to Alemayehu Shimeka; alemayehushimeka@gmail.com
}

Received 25 October 2016; Revised 3 March 2017; Accepted 10 May 2017; Published 5 June 2017

Academic Editor: Ann Duerr

Copyright (C) 2017 Maru Meseret et al. This is an open access article distributed under the Creative Commons Attribution License, which permits unrestricted use, distribution, and reproduction in any medium, provided the original work is properly cited.

\begin{abstract}
Globally, death of women due to HIV/AIDS related causes during pregnancy or within 42 days after pregnancy was estimated to be 37,000. In Ethiopia, 42,900 pregnant women living with HIV gave birth in the year 2011. This study was aimed to assess incidence and predictors of pregnancy among women on ART in Debre Markos Referral Hospital, Northwest Ethiopia. A retrospective cohort study was conducted using data recorded from September 2011 to August 2015. Data was extracted from February to March, 2016, from 1,239 records and analyzed using SPSS version 16. A Kaplan-Meier survival analysis was used to estimate the probabilities of being pregnant. The Cox proportional hazards model was done and results were expressed using hazard ratios with $95 \%$ CI. A total of 1,239 women on ART were included in the study. The incidence of pregnancy was 49.2 per 1,000 person-years. Living in rural, being married, being widowed, being unemployed, and having $<2$ children at enrollment were found to be positively associated with being pregnant. The incidence of pregnancy among women on ART was found to be considerable. Provision of family planning and other reproductive health interventions have to be coupled with the ART service to address the problem.
\end{abstract}

\section{Introduction}

An estimated 35.3 million people were living with human immune virus (HIV) worldwide, from which 3.3 million are children. Approximately, 57\% of all adults living with HIV/AIDS were women. The situation is very serious in Sub-Saharan African countries where $75 \%$ of all people with HIV in the globe are living [1]. According to UNAIDS 2013 global report, women of reproductive age in Sub-Saharan Africa were disproportionately affected by HIV/AIDS; $80 \%$ of the nearly 13 million women living with HIV worldwide live in this region [2]. According to the 2011 Ethiopian Demographic and Health Survey (EDHS), the prevalence of HIV among women aged 15-49 years was $1.9 \%$ and the prevalence increases with increasing women's age [3]. In low and middle income countries, an estimated 1.5 million women living with HIV were pregnant. Globally, death of women due to HIV/AIDS related causes during pregnancy or within 42 days after pregnancy ends was estimated to be $37,000[4]$.

Women who are living with HIV should access treatment based on WHO treatment guidelines. Nowadays, antiretroviral therapy (ART) is widely available in areas with meager resources. Providing ART for women living with HIV gives women good physical functioning and an opportunity to dream a better future. ART improves health of mothers and these mothers may be motivated to have more children [5]. In most circumstances, even though it is their right to have children, women are discouraged from getting pregnant while on ART due to the fact that giving birth from HIV positive mother could result in HIV positive babies. If proper care for the mothers is not in place, maternal death due to complications related to HIV and pregnancy can be very high [6]. 
A meta-analysis of 23 studies showed that HIV positive women have eight times risk of pregnancy related death compared to women without HIV. One in four pregnancy related deaths in Sub-Saharan Africa was attributable to HIV [7]. Despite the fact that providing treatment to women living with HIV/AIDS has a tremendous effect on the health of a mother and her newborn, a birth from HIV positive mother who is on ART may end up with not only HIV positive baby but also with maternal death. Studies have shown that, even in the presence of ART, maternal mortality rates have been reported to be five times higher in HIV infected women than in uninfected women and HIV/AIDS was responsible for at least $20 \%$ of all deaths, a figure that is higher than any direct obstetric cause [8].

A study in Uganda estimates that, even with a projected scale-up of antiretroviral therapy (ART) based prevention of mother to child transmission (PMTCT), unwanted pregnancies among women with HIV account for almost a quarter of all HIV positive infants and about a fifth of pediatric AIDS deaths [9]. HIV positive women who seek induced abortion may be at greater risk of morbidity than HIV uninfected women [10]. ART by itself may have a negative effect on birth outcomes such as prematurity or low birth weight [11].

Women living with HIV desire additional pregnancies and thus should be assisted to conceive, carry a pregnancy to term, deliver, and care for the resulting child. However, in other cases, women living with HIV become pregnant despite not desiring. Globally, many women lack access to family planning information and modern contraceptive methods; as a result, many pregnancies are unintended. In countries with the highest HIV burden, unintended pregnancies account for $14-58 \%$ of all births, and rates of unintended pregnancy among women living with HIV are high [12].

Evidences from Canada and Malawi indicated that the desire to have more children among mothers living with HIV was $69 \%$ and $17 \%$, respectively $[13,14]$. The magnitude of the desire to have more children in Ethiopia varies from place to place. The desire to have more children among mothers living with HIV in North Wollo, Addis Ababa, Harari region, and Oromia region was $15.7 \%, 44 \%, 53 \%$, and $92 \%$, respectively [15-18]. The tendency of getting pregnant and having HIV negative baby is highly improved due to the introduction of ART service which reduces mother to child transmission of HIV resulting in increased incidence of pregnancy among HIV positive women [6, 19-23].

However, because of the desire to have more children or unmet needs of family planning services, the incidence of pregnancy among women living with HIV in Ethiopia was likely to be high. Therefore, the main aim of this study was to assess the magnitude of incidence of pregnancy and predictors of it among women on HIV treatment in Debre Markos Referral Hospital.

\section{Rational of the Study}

Introduction of antiretroviral therapy to women living with HIV highly improves lifestyle and the desire to have children. However, evidences have shown that even in the presence of ART maternal mortality rates have been reported to be five times higher in HIV infected women compared to uninfected women. In Ethiopia being one of the developing countries, pregnancy related complications among women on ART are among the many health challenges. Even though the country is aggressively constructing and equipping health facilities with staff and equipment, still morbidity and mortality due to complications related to pregnancy among pregnant women on ART are very high. As far as the investigators search, there is scarcity of evidences on incidence of pregnancy among reproductive age group mothers taking ART in hospitals of Ethiopia. Considering resource limitations, this study was conducted in one of the hospitals which is Debre Markos Referral Hospital.

\section{Methods and Materials}

3.1. Study Design. Institutional based retrospective cohort study design using five-year data from September 2011 to August 2015 was employed.

3.2. Study Area and Period. This study was conducted in Debre Markos Referral Hospital. Debre Markos is a city located 300 kilometers far from Addis Ababa, the capital of Ethiopia, and $256 \mathrm{~km}$ from Bahir-Dar, a capital of Amhara National Regional State. The hospital provides health service to more than 3.5 million populations. Currently, about 100 health centers and two district hospitals are available in the catchment area of the referral hospital. There are 109 nurses, 3 health officers, 16 general practitioners, and one emergency surgeon and five specialists. Gynecologic and obstetric ward has 19 midwives, one gynecologist, and one emergency surgeon. About 8,136 patients are admitted per year, $34 \%$ of them in obstetric ward [24]. A database from I-TECH prepared excel sheet has been seen and about 1,239 women were registered on ART from September 2011 to August 2015.

3.2.1. Population. All women on ART in Debre Markos Referral Hospital catchment area were the source population for this study. All women who were on antiretroviral treatment and registered for follow-up care from September 2011 to August 2015 in the hospital were taken as the study population for this study.

3.2.2. Inclusion and Exclusion Criteria. Mothers who were getting antiretroviral treatment in the hospital from September 2011 to August 2015 were included in the study. However, mothers who were getting pre-ART care and started ART based on option $\mathrm{B}+$ and mothers with more than one pregnancy during the follow-up time in the hospital in the specified period were excluded from the study.

3.2.3. Variables. The dependent variable of the study was incidence of pregnancy which was labeled as yes and/or no. Independent variables included sociodemographic characteristics such as age, residence, marital status, occupation, educational status, number of children, and clinical characteristics like CD4 count, WHO staging, weight, BMI, and functional status of women on ART. 


\subsection{Operational Definitions}

3.3.1. Incidence of Pregnancy. In this research, incidence of pregnancy was considered as the first pregnancy after ART enrollment within the follow-up time.

3.3.2. Time to Event. In this research, time to event was defined as the time from enrollment on ART to the conception of the first pregnancy.

3.3.3. Exposed. According to this research, married women were considered as exposed to pregnancy while on ART.

3.3.4. Unexposed. Unmarried women were considered as unexposed to pregnancy while on ART.

3.3.5. Sampling Technique. The total records in the hospital among women of reproductive age group were 1,239. All the records of mothers on ART follow-up during the five consecutive years with complete information were used to determine incidence and predictors of pregnancy among women on ART.

3.3.6. Data Collection Tools. A structured data collection checklist was prepared in English to extract data from the records.

3.3.7. Data Collection Procedures. Data was extracted from main excel record of the hospital. Data elements which were not included in the excel sheet were collected from ART intake forms and cards. The data was collected by two health informatics professionals of the hospital working on ART database and supervised by one B.S. nurse.

3.3.8. Data Quality Control. Training was given for the data collectors and the supervisor before the actual data collection. Completeness of data was checked in each day of activity and the necessary feedback was offered to data collectors the next morning. Besides this, the principal investigators and an experienced data clerk carefully entered and thoroughly cleaned the data before the commencement of the analysis.

3.4. Data Management and Analysis. Date extracted from the data set was entered into excel sheet. Completeness of the excel sheet data set was checked thoroughly and exported to SPSS version 16 for analysis. Descriptive statistics and incidence rate of pregnancy in person-years of follow-up were reported. A Kaplan-Meier curve with hazard and survival functions was used to estimate the probabilities of becoming pregnant. The Cox proportional hazards model was used and results were expressed as hazard ratios with 95\% CIs. Variables with $p$ value $\leq 0.2$ at Kaplan-Meier survival analysis were entered into multivariable Cox proportional hazards model to identify the independent predictors of pregnancy. Adjusted hazard ratio with 95\% CI was used to report the findings.

3.5. Ethical Considerations. A letter of ethical clearance was obtained from Ethiopian Public Health Association and
Research Ethics Committee. Informed consent was obtained from East Gojjam Zonal Health Department and Debre Markos Referral Hospital. All data sets were extracted from ART database and stored with password. Full information was kept confidential.

\section{Results}

4.1. Sociodemographic Characteristics of Women on ART. A total of 1,951 records were reviewed. Out of these, 681 and 31 records had missing data on date of enrollment to ART and the last date of menstrual period, respectively, and thus were excluded from the analysis. Therefore, a total of 1,239 records had complete data and were considered for the final analysis. The average period for follow-up was 2.23 years with SD of 1.54 . The mean age with SD of study participants was $30 \pm 10.85$ years. Among women included in the study, 550 (44.4\%) were aged between 25 and 34 years, 957 (77.2\%) were urban dwellers, 489 (38.7\%) were married, 590 (47.6\%) were unable to read and write, $692(55.9 \%)$ have less than two children, and 975 (78.7\%) were unemployed (Table 1).

4.2. Clinical Characteristics of Women on ART. Among the study participants, $562(45.4 \%)$ had CD4 count less than 200 cells $/ \mathrm{mm}^{2}$ at enrollment, 643 (51.9\%) had weight less than 50 kilograms, 440 (35.5\%) were at 3rd clinical stage, 908 (73.3\%) had a BMI of 18.5 to 25 , and $1,030(84.2 \%)$ of them had a functional status of working at ART enrollment (Table 2).

4.3. Incidence of Pregnancy. Initially, a total of 1,239 women on ART were included in the study from September 2011 to August 2015 at different entry to the cohorts. The highest number of enrollment to ART was registered during 2011 which was $355(28.7 \%)$. The overall incidence of pregnancy was found to be 49.2 per 1000 person-years (136 pregnancies in a total of 2762.55 years of observation). The incidence of pregnancy among exposed women was 74.6 per 1000 person-years ( 79 pregnancies in a total of 1058.88 years of observation) while it was 33.46 per 1000 person-years (57 pregnancies in a total of 1703.67 years of observation) in unexposed. The highest rate of pregnancy was registered among the cohorts of 2015 with 109.5 per 1000 person-years.

4.4. Kaplan-Meier Survival Analysis. The cumulative probability of surviving from pregnancy was higher among women aged 35 and above with mean survival time 4.60 years, $95 \% \mathrm{CI}$ (4.47-4.74). This means the cumulative hazard of pregnancy was higher among women aged 25 to 34 years (Figure 1). At Kaplan-Meier analysis, statistically significant association was observed with incidence of pregnancy at $p$ value $<0.05$. At the beginning of the study, the probability of getting pregnant for all women was almost the same. However, the risk of getting pregnant among women having less than two children was increasing with follow-up time. The cumulative probability of surviving from pregnancy for all women was almost the same, but after follow-up it was higher among women having two and above children than those having less than two children with mean survival time of 4.56 years, $95 \%$ CI (4.44-4.68) and 4.33, 95\% CI (4.20-4.46), respectively. 
TABLE 1: Sociodemographic characteristics of women receiving ART treatment from Sep. 2011 to Aug. 2015 in Debre Markos Referral Hospital, Northwest Ethiopia, $2016(N=1239)$.

\begin{tabular}{|c|c|c|c|}
\hline \multirow{2}{*}{ Variables } & \multicolumn{2}{|c|}{ Pregnancy status } & \multirow{2}{*}{ Total frequency } \\
\hline & No & Yes & \\
\hline \multicolumn{4}{|l|}{ Age } \\
\hline 15-24 years & $267(89.3 \%)$ & $32(10.7 \%)$ & $299(24.1 \%)$ \\
\hline $25-34$ years & $476(86.5 \%)$ & $74(13.5 \%)$ & $550(44.4 \%)$ \\
\hline$\geq 35$ years & $360(92.3 \%)$ & $30(7.7 \%)$ & $390(31.5 \%)$ \\
\hline \multicolumn{4}{|l|}{ Place of residence } \\
\hline Urban & $861(90 \%)$ & $96(10 \%)$ & $957(77.2 \%)$ \\
\hline Rural & $242(85.8 \%)$ & $40(14.2 \%)$ & $282(22.8 \%)$ \\
\hline \multicolumn{4}{|l|}{ Marital status } \\
\hline Never married & $239(95.2 \%)$ & $12(4.8 \%)$ & $251(20.3 \%)$ \\
\hline Married & $400(83.5 \%)$ & $79(16.5 \%)$ & $479(38.7 \%)$ \\
\hline Widowed & $258(91.5 \%)$ & $24(8.5 \%)$ & $282(22.8 \%)$ \\
\hline Divorced & $206(90.7 \%)$ & $21(9.3 \%)$ & $227(18.3 \%)$ \\
\hline \multicolumn{4}{|l|}{ Educational status } \\
\hline Unable to read and write & $493(83.6 \%)$ & $97(16.4 \%)$ & $590(47.6 \%)$ \\
\hline Primary $(1-8)$ & $224(94.5 \%)$ & $13(5.5 \%)$ & $237(19.1 \%)$ \\
\hline Secondary (9-12) & $197(92.1 \%)$ & $17(7.9 \%)$ & $214(17.3 \%)$ \\
\hline Certificate and above & $189(95.5 \%)$ & $9(4.5 \%)$ & $198(16 \%)$ \\
\hline \multicolumn{4}{|l|}{ Occupational status } \\
\hline Unemployed & $858(88 \%)$ & $117(12 \%)$ & $975(78.7 \%)$ \\
\hline Employed & $245(92.8 \%)$ & $19(7.2 \%)$ & $264(21.3 \%)$ \\
\hline \multicolumn{4}{|c|}{ Number of children at enrollment } \\
\hline $0-1$ child & $604(87.3 \%)$ & $88(12.7 \%)$ & $692(55.9 \%)$ \\
\hline$\geq 2$ children & $499(91.2 \%)$ & $48(8.8 \%)$ & $547(44.1 \%)$ \\
\hline
\end{tabular}

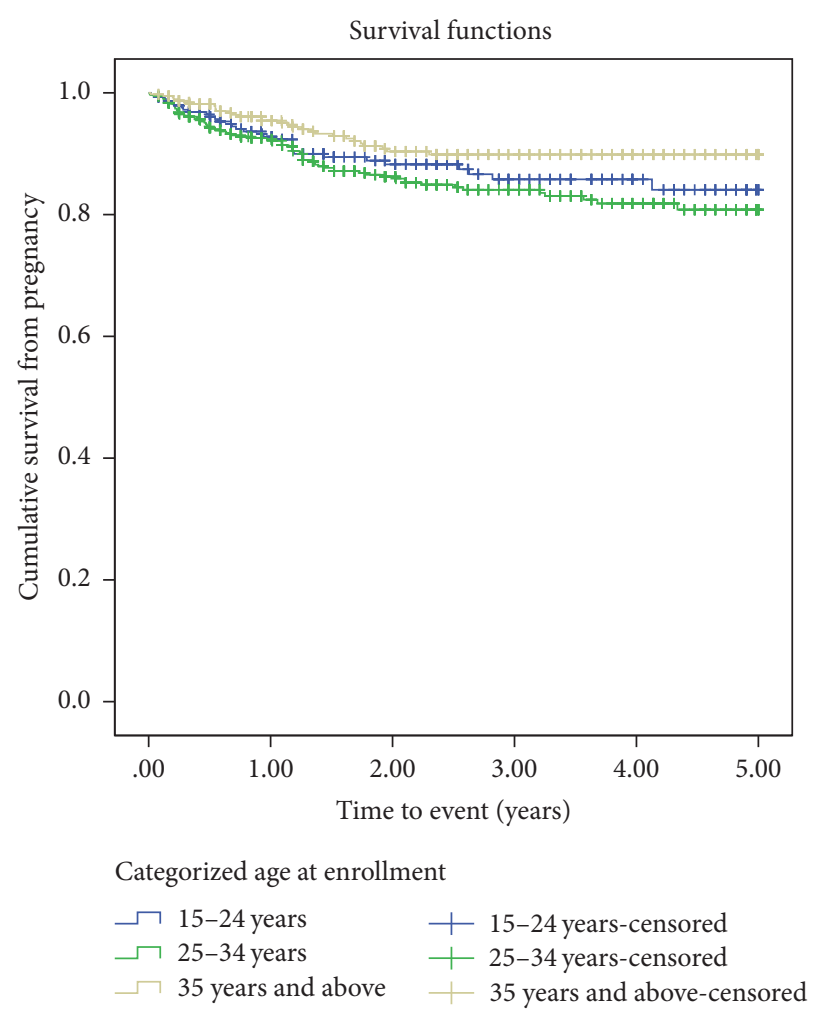

FIGURE 1: Kaplan-Meier curves of pregnancy comparing age among women receiving ART treatment from Sep. 2011 to Aug. 2015 in Debre Markos Referral Hospital, Northwest Ethiopia, $2016(N=1,239)$. 
TABLE 2: Clinical characteristics of women receiving ART treatment in Debre Markos Referral Hospital, Northwest Ethiopia, Sep. 2011 to Aug. $2015(N=1239)$.

\begin{tabular}{|c|c|c|}
\hline \multirow{2}{*}{ Variables } & \multicolumn{2}{|c|}{ Pregnancy status } \\
\hline & No & Yes \\
\hline \multicolumn{3}{|c|}{ CD4 count at enrollment } \\
\hline$<200 \mathrm{cell} / \mathrm{mm}^{2}$ & $488(86.8 \%)$ & $74(13.2 \%)$ \\
\hline $200-350 \mathrm{cell} / \mathrm{mm}^{2}$ & $446(91 \%)$ & $44(9 \%)$ \\
\hline$>350 \mathrm{cell} / \mathrm{mm}^{2}$ & $169(90.4 \%)$ & $18(9.6 \%)$ \\
\hline \multicolumn{3}{|c|}{ Functional status at enrollment } \\
\hline Working & $913(88.6 \%)$ & $117(11.4 \%)$ \\
\hline Ambulatory & $163(91.6 \%)$ & $15(8.4 \%)$ \\
\hline Bedridden & $15(100 \%)$ & $0(0 \%)$ \\
\hline \multicolumn{3}{|l|}{ Weight at enrollment } \\
\hline$\leq 50 \mathrm{~kg}$ & $580(90.2 \%)$ & $63(9.8 \%)$ \\
\hline $51-60 \mathrm{~kg}$ & $307(89 \%)$ & $38(11 \%)$ \\
\hline $61-70 \mathrm{~kg}$ & $100(84 \%)$ & $19(16 \%)$ \\
\hline$\geq 71 \mathrm{~kg}$ & $116(87.9 \%)$ & $16(12.1 \%)$ \\
\hline \multicolumn{3}{|c|}{ WHO clinical stage at enrollment } \\
\hline Clinical stage 1 & $367(89.5 \%)$ & $43(10.5 \%)$ \\
\hline Clinical stage 2 & $258(85.7 \%)$ & $43(14.3 \%)$ \\
\hline Clinical stage 3 & $395(89.8 \%)$ & $45(10.2 \%)$ \\
\hline Clinical stage 4 & $83(94.3 \%)$ & $5(5.7 \%)$ \\
\hline \multicolumn{3}{|l|}{ BMI at enrollment } \\
\hline$<18.5$ & $127(91.4 \%)$ & $12(8.6 \%)$ \\
\hline $18.5-25$ & $802(88.3 \%)$ & $106(11.7 \%)$ \\
\hline$>25$ & $174(90.6 \%)$ & $18(9.4 \%)$ \\
\hline
\end{tabular}

At Kaplan-Meier analysis, statistically significant association was observed with incidence of pregnancy at $p$ value $<0.05$ (Figure 2).

With respect to residence, the cumulative hazard of pregnancy at year one for urban and rural residents was $5 \%$ and $9 \%$, respectively, whereas the cumulative probability of surviving at year one for urban and rural residents was $89 \%$ and $85 \%$ with mean survival time 4.48 years, $95 \%$ CI [4.38-4.57], and 4.28 years, 95\% CI [4.08-4.49], respectively. The cumulative hazard of pregnancy at year one for women with marital status never married, married, widowed, and divorced was $4 \%, 9 \%, 6 \%$, and $3 \%$, respectively, whereas the cumulative probability of surviving at year one for women with marital status never married, married, widowed, and divorced was $94 \%, 82 \%, 91 \%$, and 90\%, respectively. At Kaplan-Meier analysis, statistically significant association was observed with incidence of pregnancy at $p$ value $<$ 0.05. The lowest cumulative probability of surviving from pregnancy had been observed among women with marital status married in the remaining years. Throughout followup period, the risk of getting pregnant was increasing among women who were unable to read and write compared to those with primary and above education. At Kaplan-Meier analysis, statistically significant association was observed with incidence of pregnancy at $p$ value $<0.05$. Cumulative probability of getting pregnant among unemployed mothers was higher during early phase of the study and continued to increase throughout follow-up years compared to employed mothers whereas the cumulative probability of surviving from pregnancy among employed mothers is higher than unemployed with mean survival time of 4.63 years, $95 \% \mathrm{CI}$ (4.46-4.78), and 4.38 years, 95\% CI (4.28-4.48), respectively. At Kaplan-Meier estimate, statistically significant association was observed with incidence of pregnancy at $p$ value $<0.05$.

4.5. Predictors of Pregnancy among Mothers on ART. Variables with a $p$ value of $<0.2$ at Kaplan-Meier estimate were used to assess the actual effect on incidence of pregnancy. Backward stepwise method was used. The risk of getting pregnant among rural residents women was 17.87 times higher compared to urban counterparts $[\mathrm{AHR}=17.87,95 \% \mathrm{CI}$ (10.72-29.79)]. Married, widowed, and divorced women had $5.35,3.65$, and 4.34 times higher risk of pregnancy compared to women who had never married [AHR $=5.35,95 \% \mathrm{CI}$ (2.14-13.36), AHR = 3.65, 95\% CI (1.06-12.49), and AHR $=4.34,95 \%$ CI (1.42-13.22), resp.]. Women who had less than two children had 2.05 times higher risk of pregnancy compared to those who had two or more children [AHR $=2.05,95 \%$ CI (1.31-3.20)]. Unemployed women had 4.37 times higher incidence of pregnancy compared to employed counterparts $[\mathrm{AHR}=4.37,95 \% \mathrm{CI}$ (1.91-9.98)] (Table 3).

\section{Discussion}

This study has aimed at assessing the incidence of pregnancy and its predictors. Accordingly, the overall incidence of 


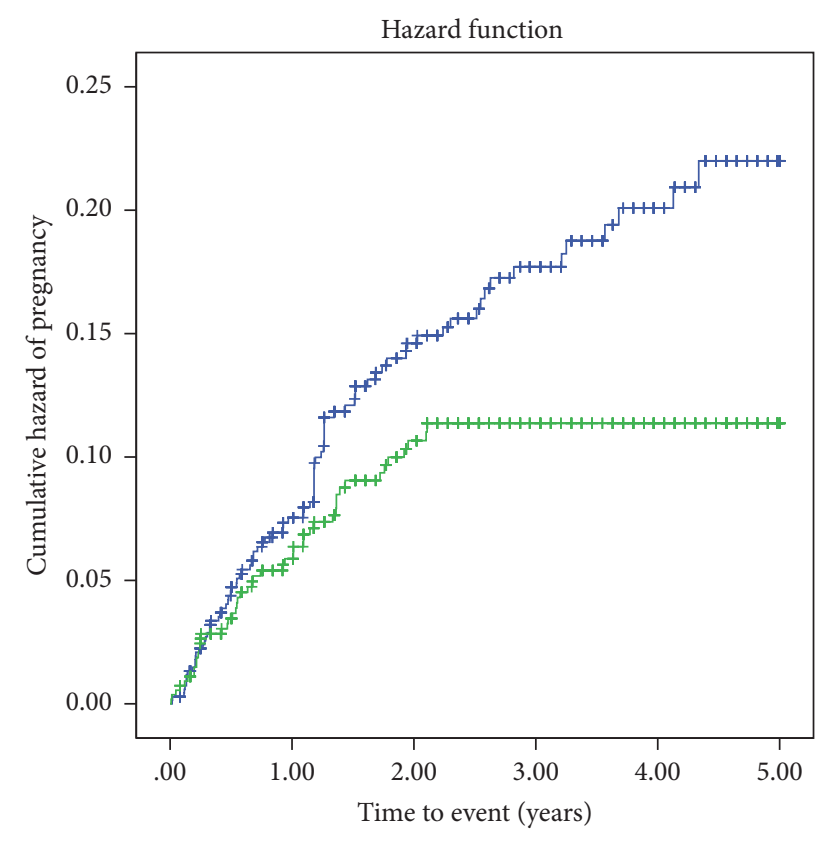

Number of children at enrollment

$\begin{array}{ll}\neg 0 \text { - } 1 \text { child } & +0 \text { - } 1 \text { child-censored } \\ \neg>+2 \text { children } & +>+2 \text { children-censored }\end{array}$

FIGURE 2: Kaplan-Meier curves of pregnancy showing hazard of pregnancy among women receiving ART treatment from Sep. 2011 to Aug. 2015 in Debre Markos Referral Hospital, Northwest Ethiopia, $2016(N=1,239)$.

pregnancy was found to be 49.2 per 1000 person-years. This finding was lower than the findings of other studies such as United States of America [6], Sub-Saharan Africa [25], Uganda, and Ethiopia [26-30] with 74, 78, 90.7, and 65 per 1000 person-years, respectively.

The possible reasons for the explanation might be due to differences in the access and quality of the services, the size of records reviewed, the study period, and differences in inclusion and exclusion criteria. The risk of getting pregnant was 17.87 times higher among the rural women compared to their urban counterparts. This could be due to the fact that, in rural areas, the accessibility of family planning services was lower in the community and for mothers on ART in particular.

Married, widowed, and divorced women were 5.35, 3.65, and 4.34 times higher compared to women who never married, respectively. This finding was in line with the findings from United States of America and Sub-Saharan Arica $[6,25]$; however, it was not consistent with the findings of a study of Western Uganda [26]. The possible explanation for the discrepancies might be due to differences in the size of records reviewed, the study period, and differences in inclusion and exclusion criteria.

The risk of pregnancy was 2.05 times higher among mothers who had zero or one child compared to those who had two and above children. This finding was in line with the findings of a study done in Western Uganda and Ethiopia [26, 30].
Women who had less than two children might have high fertility desire compared to those having two or more children. This might be due to the fact that mothers who had two or more children had better utilization of family planning services. The risk of pregnancy among unemployed women was also 4.37 times higher compared to the employed. It was in line with the findings of a study done in Western Uganda [26]. The possible justification of this finding might be due to the fact that in most circumstances in Ethiopia employed women are those with better educational status; in turn those women having better education may have a chance to access information from different types of media.

\section{Limitations of the Study}

Since this research was done using secondary data, all variables such as disclosure to sexual partner, adherence to ART, family planning methods uptake, open discussion with sexual partner, and other reproductive health issues which can possibly affect pregnancy among women on ART were not included. In addition to this, excluding charts with incomplete records could have an effect on the validity of the findings.

\section{Conclusion}

In light of the findings of this study, the authors forwarded the following remarks. The incidence of pregnancy among women on ART in Debre Markos Referral Hospital was found to be considerable. Living in rural areas, being married, widowed, and divorced, being unemployed, and having less than two children at enrollment on ART were found to be predictors of incidence of pregnancy among women on ART.

\section{Recommendation}

Considering the findings of this study, the Regional Health Bureau, Zonal Health Department, and health institutions should ensure that reproductive health services have to be well integrated into the HIV care system. Effective counseling strategies have to be designed focusing on rural, married, widowed, and divorced, unemployed mothers and mothers with less than two children at enrollment to reduce the risks of getting pregnant.

\section{Conflicts of Interest}

The authors have declared that they have no conflicts of interest.

\section{Authors' Contributions}

Maru Meseret conceptualized and designed the study, developed tools, supervised data collection, performed analysis and interpretation of data, and drafted the paper. Alemayehu Shimeka assisted in the conception and design of the study and helped in writing the proposal, developing tools, performing the analysis, and writing the paper. He has also developed the manuscript. Alemayehu Bekele assisted in the 
TABLE 3: Cox's proportional hazards models examining predictors of incidence of pregnancy with 95\% CIs, among women on ART in Debre Markos Referral Hospital, Northwest Ethiopia, Sept. 2011 to Aug. $2015(N=1,239)$.

\begin{tabular}{|c|c|c|c|c|}
\hline \multirow{2}{*}{ Variable } & \multicolumn{2}{|c|}{ Status of pregnancy } & \multirow{2}{*}{ CHR (95\% CI) } & \multirow{2}{*}{$\operatorname{AHR}(95 \% \mathrm{CI})$} \\
\hline & No & Yes & & \\
\hline \multicolumn{5}{|l|}{ Place of residence } \\
\hline Urban & $861(90 \%)$ & $96(10 \%)$ & 1 & 1 \\
\hline Rural & $242(85.8 \%)$ & $40(14.2 \%)$ & $1.40(0.97-2.02)$ & $17.87(10.72-29.79)$ \\
\hline \multicolumn{5}{|l|}{ Age } \\
\hline $15-24$ & $267(89.3 \%)$ & $32(10.7 \%)$ & 1 & \\
\hline $25-34$ & $476(86.5 \%)$ & $74(13.5 \%)$ & $1.22(0.81-1.85)$ & \\
\hline$\geq 35$ years & $360(92.3 \%)$ & $30(7.7 \%)$ & $0.68(0.41-1.12)$ & \\
\hline \multicolumn{5}{|l|}{ Marital status } \\
\hline Never married & $239(95.2 \%)$ & $12(4.8 \%)$ & 1 & 1 \\
\hline Married & $400(83.5 \%)$ & $79(16.5 \%)$ & $3.61(1.97-6.64)$ & $5.35(2.14-13.36)$ \\
\hline Widowed & $258(91.5 \%)$ & $24(8.5 \%)$ & $1.81(0.90-3.62)$ & $3.65(1.06-12.49)$ \\
\hline Divorced & $206(90.7 \%)$ & $21(9.3 \%)$ & $2.13(1.05-4.34)$ & $4.34(1.42-13.22)$ \\
\hline \multicolumn{5}{|l|}{ Educational status } \\
\hline Unable to read \& write & $493(83.6 \%)$ & $97(16.4 \%)$ & $3.83(1.93-7.59)$ & $2.28(0.89-5.84)$ \\
\hline Primary $(1-8)$ & $224(94.5 \%)$ & $13(5.5 \%)$ & $1.24(0.53-2.90)$ & $0.77(0.24-2.48)$ \\
\hline Secondary (9-12) & $197(92.1 \%)$ & $17(7.9 \%)$ & $1.81(0.80-4.06)$ & $1.31(0.37-4.61)$ \\
\hline Certificate and above & $189(95.5 \%)$ & $9(4.5 \%)$ & 1 & 1 \\
\hline \multicolumn{5}{|l|}{ Occupational status } \\
\hline Unemployed & $858(88 \%)$ & $117(12 \%)$ & $1.72(1.06-2.80)$ & 4.37 (1.91-9.98) \\
\hline Employed & $245(92.8 \%)$ & $19(7.2 \%)$ & 1 & 1 \\
\hline \multicolumn{5}{|c|}{ Number of children at enrollment } \\
\hline 0-1 child & $604(87.3 \%)$ & $88(12.7 \%)$ & $1.55(1.09-2.21)$ & $2.05(1.31-3.20)$ \\
\hline$\geq 2$ children & $499(91.2 \%)$ & $48(8.8 \%)$ & 1 & 1 \\
\hline
\end{tabular}

Note: level of significance was considered at a cutoff point of the $p$ value of 0.05 .

conception, design, and interpretation of results and drafting the manuscript. All the authors read and approved the final manuscript.

\section{Acknowledgments}

The authors would like to acknowledge Ethiopian Public Health Association (EPHA) for the financial support to conduct this study. They are indebted to the administration of Debre Markos Referral Hospital for allowing them to access the data. They would like to thank also the study participants.

\section{References}

[1] HIV/AIDS, "Global fact sheet: stepping up the pace," in Proceedings of the 20th International AIDS Conference, Melbourne, Australia, July 2014.

[2] Joint United Nations Programs on HIV/AIDS, "Global Report: UNAIDS Report on the Global AIDS Epidemic," Tech. Rep., UNAIDS, Geneva, Switzerland, 2013.

[3] Central Statistical Agency and ICF International, "Ethiopia Demographic and Health Survey 2011," Tech. Rep., Central Statistical Agency and ICF International, Addis Ababa, Ethiopia, 2012.

[4] UNAIDS, "A progress report on the Global Plan towards the elimination of new HIV infections among children by 2015 and keeping their mothers alive, 2012".
[5] R. P. Walensky and D. R. Kuritzkes, "The impact of the President's Emergency Plan for AIDS Relief (PEPfAR) beyond HIV and why it remains essential," Clinical Infectious Diseases, vol. 50, no. 2, pp. 272-275, 2010.

[6] L. S. Massad, G. Springer, L. Jacobson et al., "Pregnancy rates and predictors of conception, miscarriage and abortion in US women with HIV," AIDS, vol. 18, no. 2, pp. 281-286, 2004.

[7] C. Calvert and C. Ronsmans, "The contribution of HIV to pregnancy-related mortality: a systematic review and metaanalysis," AIDS, vol. 27, no. 10, pp. 1631-1639, 2013.

[8] G. E. Gray and J. A. McIntyre, "HIV and pregnancy," British Medical Journal, vol. 334, no. 7600, pp. 950-953, 2007.

[9] W. Hladik, J. Stover, G. Esiru, M. Harper, and J. Tappero, "The contribution of family planning towards the prevention of vertical HIV transmission in Uganda," PLoS ONE, vol. 4, no. 11, article e7691, 2009.

[10] M. De Bruyn, "Safe abortion for HIV-positive women with unwanted pregnancy: a reproductive right," Reproductive Health Matters, vol. 11, no. 22, pp. 152-161, 2003.

[11] D. K. Ekouevi, P. A. Coffie, R. Becquet et al., "Antiretroviral therapy in pregnant women with advanced HIV disease and pregnancy outcomes in Abidjan, Côte d'Ivoire," AIDS, vol. 22, no. 14 , pp. 1815-1820, 2008.

[12] H. W. Reynolds, B. Janowitz, R. Wilcher, and W. Cates, "Contraception to prevent HIV-positive births: current contribution and potential cost savings in PEPFAR countries," Sexually Transmitted Infections, vol. 84, (Suppl 2): ii49-53, no. 2, 2008. 
[13] M. R. Loutfy, T. A. Hart, S. S. Mohammed et al., "Fertility desires and intentions of HIV-positive women of reproductive age in Ontario, Canada: a cross-sectional study," PLoS ONE, vol. 4, no. 12, article e7925, 2009.

[14] A. L. N. Dube, A. Baschieri, J. Cleland et al., "Fertility intentions and use of contraception among monogamous couples in northern malawi in the context of hiv testing: a cross-sectional analysis," PLoS ONE, vol. 7, no. 12, article e51861, 2012.

[15] M. Getachew, F. Alemseged, M. Abera, and A. Deribew, "Factors affecting fertility decisions of married men and women living with HIV in South Wollo Zone, Northeast Ethiopia," Ethiopian Journal of Health Development, vol. 24, no. 3, pp. 204-220, 2010.

[16] H. M. Asfaw and F. E. Gashe, "Fertility intentions among HIV positive women aged 18-49 years in Addis Ababa Ethiopia: a cross sectional study," Reproductive Health, vol. 11, no. 1, article 36, 2014.

[17] F. Haile, N. Isahak, and A. Dessie, "Fertility desire and associated factors among people living with HIV on ART, in Harari Regional State, Eastern Ethiopia," Journal of Tropical Diseases, vol. 2, article 2, 2014.

[18] Y.-T. Bonnenfant, M. J. Hindin, and D. Gillespie, "HIV diagnosis and fertility intentions among couple VCT clients in Ethiopia," AIDS Care, vol. 24, no. 11, pp. 1407-1415, 2012.

[19] C. Kilewo, K. Karlsson, M. Ngarina et al., "Prevention of mother-to-child transmission of HIV-1 through breastfeeding by treating mothers with triple antiretroviral therapy in dar es salaam, tanzania: the mitra plus study," Journal of Acquired Immune Deficiency Syndromes, vol. 52, no. 3, pp. 406-416, 2009.

[20] T. K. Thomas, R. Masaba, C. B. Borkowf et al., "Tripleantiretroviral prophylaxis to prevent mother-to-child HIV transmission through breastfeeding-the Kisumu Breastfeeding Study, Kenya: a clinical trial," PLoS Medicine, vol. 8, no. 3, Article ID e1001015, 2011.

[21] N. Siegfried, L. van der Merwe, P. Brocklehurst, and T. T. Sint, "Antiretrovirals for reducing the risk of mother-to-child transmission of HIV infection," Cochrane Database of Systematic Reviews, no. 7, 2011.

[22] J. J. C. Lewis, C. Ronsmans, A. Ezeh, and S. Gregson, "The population impact of HIV on fertility in Sub-Saharan Africa," AIDS, vol. 18, no. 2, pp. S35-S43, 2004.

[23] B. Zaba and S. Gregson, "Measuring the impact of HIV on fertility in Africa," AIDS, vol. 12, no. 1, pp. S41-S50, 1998.

[24] M. Gedefaw, H. Gebrehana, A. Gizachew, and F. Taddess, "Assessment of maternal near miss at debre markos referral hospital, northwest ethiopia: five years experience," Open Journal of Epidemiology, vol. 4, pp. 199-207, 2014.

[25] L. Myer, R. J. Carter, M. Katyal, P. Toro, W. M. El-Sadr, and E. J. Abrams, "Impact of antiretroviral therapy on incidence of pregnancy among HIV-infected women in Sub-Saharan Africa: a cohort study," PLoS Medicine, vol. 7, no. 2, Article ID e1000229, 2010.

[26] J. Kabami, E. Turyakira, S. Biraro, and F. Bajunirwe, "Increasing incidence of pregnancy among women receiving HIV care and treatment at a large urban facility in western Uganda," Reproductive Health, vol. 11, article 81, 2014.

[27] J. Homsy, R. Bunnell, D. Moore et al., "Reproductive intentions and outcomes among women on antiretroviral therapy in rural Uganda: a prospective cohort study," PLoS ONE, vol. 4, no. 1, Article ID e4149, 2009.

[28] A. Kaida, L. T. Matthews, S. Kanters et al., "Incidence and predictors of pregnancy among a cohort of HIV-positive women initiating antiretroviral therapy in mbarara, Uganda," PLoS ONE, vol. 8, no. 5, Article ID e63411, 2013.

[29] F. E. Makumbi, G. Nakigozi, S. J. Reynolds et al., "Associations between HIV antiretroviral therapy and the prevalence and incidence of pregnancy in Rakai, Uganda," AIDS Research and Treatment, vol. 2011, Article ID 519492, p. 10, 2011.

[30] M. Degife, G. Degu, and M. Molla, "Assessment of incidence and predictors of pregnancy among patients on antiretroviral therapy (ART) and pre-ART: a retrospective cohort study," The Lancet, vol. 381, article S34, 2013. 


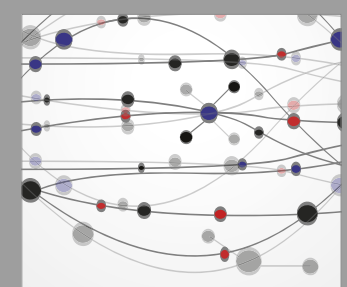

The Scientific World Journal
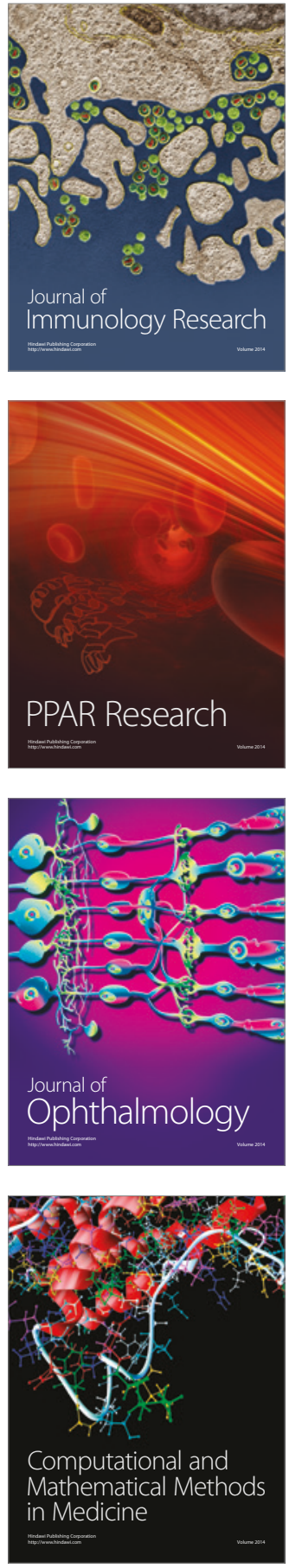

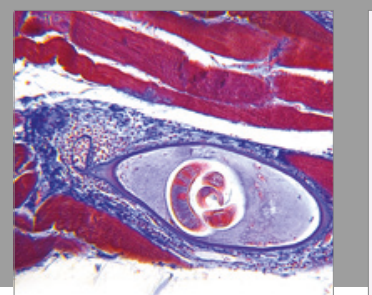

Gastroenterology Research and Practice
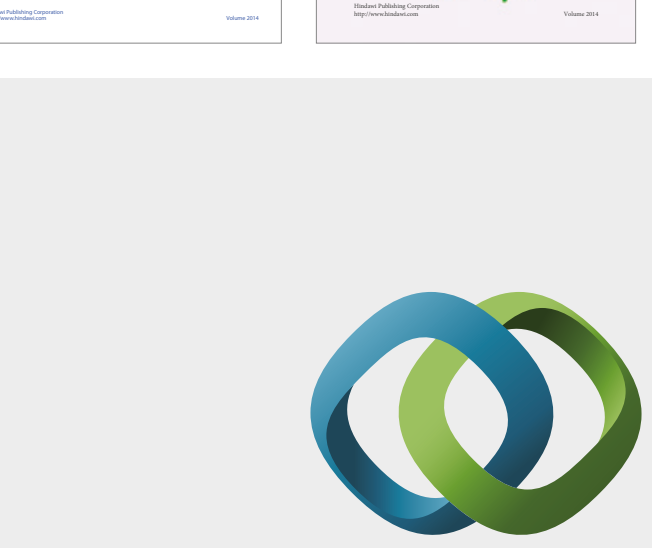

\section{Hindawi}

Submit your manuscripts at

https://www.hindawi.com
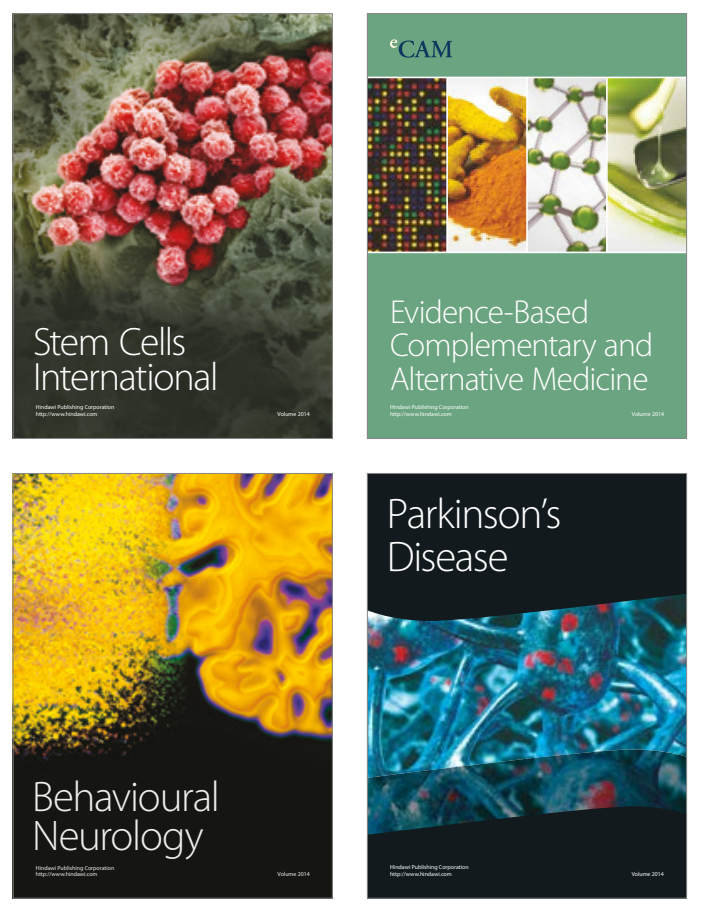
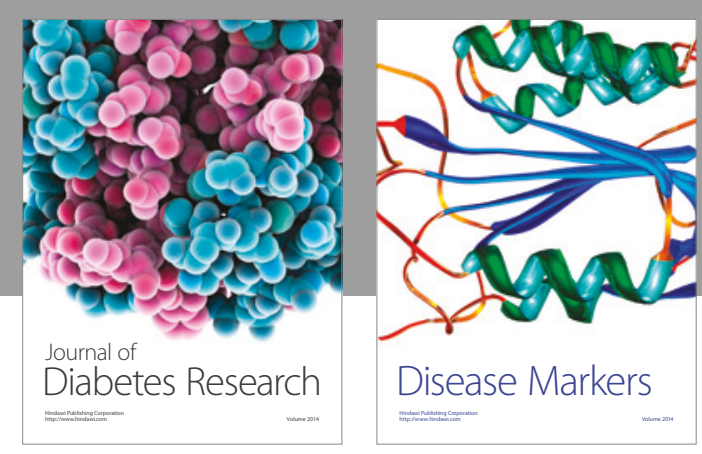

Disease Markers
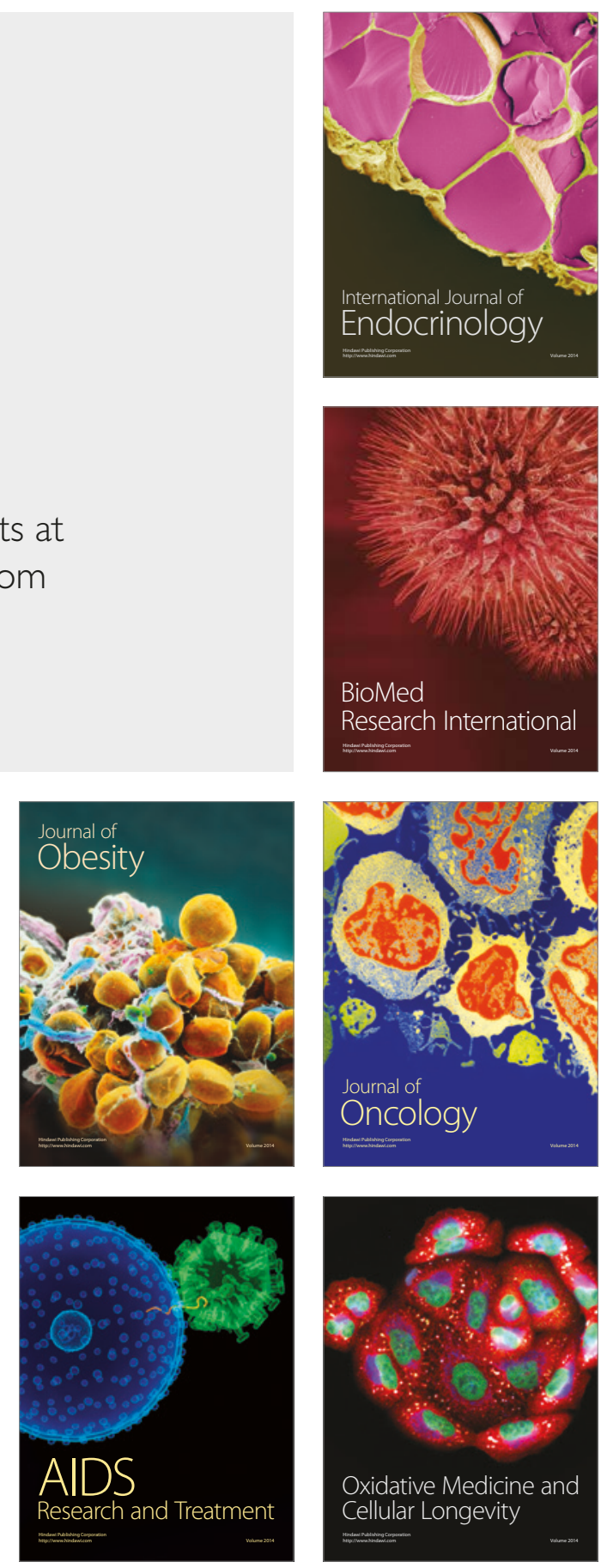